Results Five studies involving 73 patients were included in this review. Four studies (59-subjects) compared the efficacy of nebulisers against MDI, and one study (14-subjects) compared ELB against MDI. The review found that all three modes were effective in significantly reducing both PIP and $R_{a w}$, with two studies suggesting that nebulisers appear to be more effective than MDI (GRADE - moderate). ELB was found to be especially effective as a rescue therapy when conventional management had failed (GRADE - moderate). The studies were limited by small sample sizes, large variability in outcomes measures, incomplete reporting and a high degree of heterogeneity; thus, precluding a meta-analysis. The risk of bias ranged from low to uncertain across most domains.

Conclusions A systematic review of RCTs found that there was insignificant evidence to assert the superiority of any one mode of bronchodilators over the other, and thus a balanced and nuanced approach to managing acute bronchospasm should be contextualised to the individual needs and bestinterest of the patient using a multi-modal approach. Further high-quality RCTs with larger samples sizes, preferably comparing all three modalities are required to conclusively provide a tangible answer.

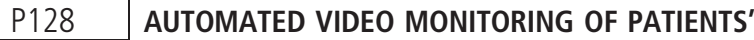 SPONTANEOUS BREATHING DURING HIGH FREQUENCY JET VENTILATION}

${ }^{1} \mathrm{MB}$ Kontorovich, ${ }^{2} \mathrm{KS}$ Purtov, ${ }^{2} \mathrm{VS}$ Kublanov. ${ }^{1}$ Regional Anti-Tuberculous Dispensary, Yekaterinburg, Russia; ${ }^{2}$ Ural Federal University, Engineering School of Information Technologies Telecommunications and Control Systems, Yekaterinburg, Russia

\subsection{6/thoraxjnl-2017-210983.270}

Introduction One important problem in anaesthetic equipment is the measurement of patients' spontaneous breathing during artificial ventilation in the immediate postoperative period. The current paper presents an investigation into using video processing technology to determine the accuracy of spontaneous breathing evaluation for patients during high frequency jet ventilation (HFJV). We call this technology remote body plethysmography(rBPG).

Materials and Methods The 6 patients (male and female) involved, aged between 42 and 70, had undergone operation of the thoracic cavity. Each patient provided written informed voluntary consent prior to study procedures. In the immediate postoperative period, patients enter the intensive care unit, and HFJV is administered for a time between $30 \mathrm{~min}$ up to 2 hours or until full reestablishment of adequate spontaneous breathing is made. The HFJV procedure was performed by the ZisLine JV100A device (Triton Electronics Systems Ltd., Russia, registration No: 2010/08739). The reference impedance pneumography signal was provided by an MP 6-03 monitor (Triton Electronics Systems Ltd., Russia, registration No: 2007/00597). The video recording was performed at a distance of $80 \mathrm{~cm}$. Low-cost Logitech C920 webcams with $30 \mathrm{~Hz}$ sampling frequency and a $640 \times 480$ pixel resolution were used. The rBPG signal was measured in real-time with the use of original software.

Results According to rBPG the mean error of respiration rate estimation did not exceed $0.053 \mathrm{~Hz}(3.2$ breath per minute) with a standard deviation of less than $0.04 \mathrm{~Hz}$ (2.45 breaths per minute). The example of respiration rate estimation presented in figure 1 . In 4 cases, the rBPG and impedance pneumography breath frequency evaluation started at the same

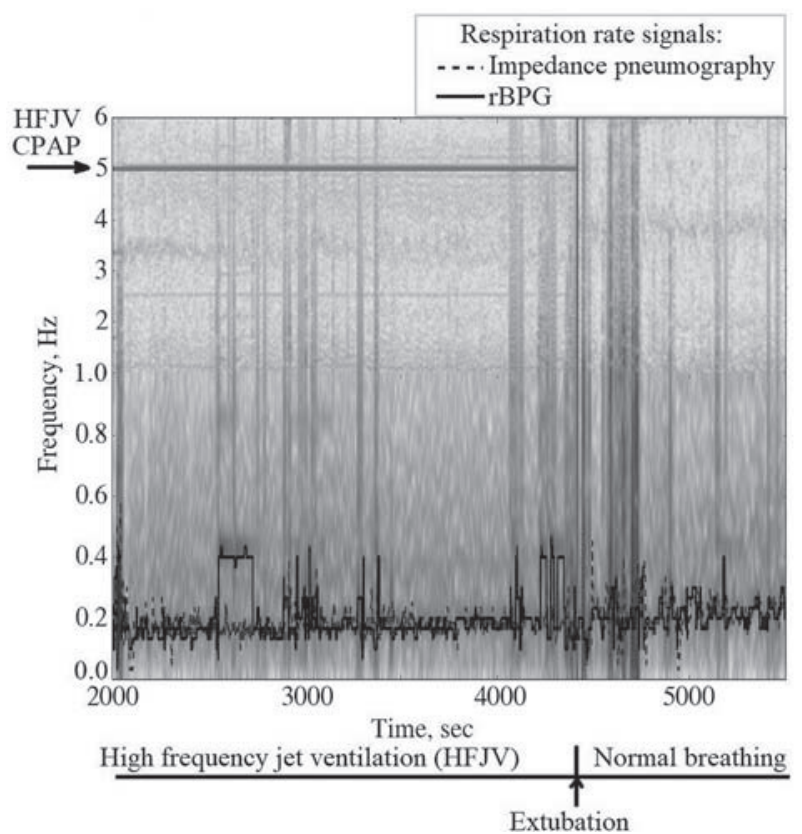

Abstract P128 Figure 1 rBPG signal spectrum with examples of respiration rate estimation during the HFJV.

time. In 2 cases the rBPG frequency estimation started more than 1 min earlier.

Conclusion rBPG provides reliable and early determination of spontaneous breathing during HFJV. Therefore, it can be recommended as an additional method of non-invasive spontaneous breathing monitoring to improve the safety of patients during HFJV.

\section{P129 IMPLEMENTING TARGET RANGE OXYGEN IN CRITICAL CARE (TROCC); A BASELINE SURVEY AND PILOT STUDY}

BR O'Driscoll, T Fudge, J Cardell, H Millar, PM Dark. Salford Royal Foundation NHST, Salford, UK

\subsection{6/thoraxjn|-2017-210983.271}

Iatrogenic hyperoxaemia is common on Critical Care Units (CCUs) throughout the world and high blood oxygen levels have been associated with adverse outcomes including increased mortality. We have commenced a pilot quality implementation study to analyse the views of Critical Care staff regarding oxygen therapy and to change practice to ensure that all patients in the Critical Care Unit have a prescribed target oxygen saturation range. $33 \mathrm{CCU}$ staff responded to an online questionnaire (16 doctors, 7 nurses, 9 physiotherapists, 1 ACCP). 76\% thought that slightly too much oxygen was used on the unit but only $53 \%$ favoured a formal prescription for oxygen for all patients. For ventilated patients not at risk of hypercapnia, $83 \%$ would favour a target range of 94\%$98 \%$ and $10 \%$ would opt for a target range of 90\%-94\%. For patients at risk of hypercapnia, all respondents favoured a target range of $88 \%-92 \%$. A baseline audit of practice on the unit studied 54 patients (28 on ventilators) over one month prior to the implementation of a programme of change. $85 \%$ of audited patients (46 of 54) had a formal oxygen prescription with target range. Forty patients had target range 94\%$98 \%$ and six patients had target range $88 \%-92 \%$, all 